\title{
Open Space Experimental Work for L-Band Mobile Satellite (MS) using a Simple and Low Cost Data Acquisition System
}

\author{
I. Abba, W.A. Wan Zainal Abidin, V.P. Bong, A.K. Othman, K. Hong Ping \\ Department of Electronic Engineering, \\ Universiti Malaysia Sarawak, 94300 Kota Samarahan, Sarawak, Malaysia.
}

\begin{abstract}
Mobile satellite (MS) signal performance is affected by many factors such as ionospheric effect, multipath fading and shadowing effect. Satellites transmit signals at the same strength, but things like trees and buildings can obscure a signal beyond recognition. Some experimental works have been done using the existing geo-satellite system or airborne platform in the developed countries which is quite expensive and complicated. Very little data is represents less developed countries such as Africa and Asia. Therefore, a simple and low cost data acquisition system that can be used to carry out measurement of the MS signal performance is proposed. The experiment is carried out in open area with no obstruction of the direct wave. In addition, NMEA sentences will be used to obtain the required signal propagation parameters. The measurement is then performed to determine the relationship between the signal performance with respect to the elevation and azimuth angles. Results obtained from analysis will be used to determine the characteristics of the open space environment. This method can be used to encourage more experimental works in the less develop countries. Propagation data for the open space environment can be used as a reference to determine the MS signal quality for shadowing environments.
\end{abstract}

Keywords- Mobile satellite(MS); Hand held GPS receiver; Open space; Cost-effective;

\section{INTRODUCTION}

Mobile Satellites (MS) in communication system has become a vital part in human daily life as these can be seen from the amount of antennas or parabolic dishes which are fixed at many homes for the television broadcast service. Besides, satellite also play an essential part such as navigation and position allocation, terrain observation, weather monitoring, deep-space exploration, remote sensing and others.

Communication satellites function as a microwave repeater station for the exchanging of the information between the users in different forms [1]. However GPS (Global Positioning System) is best known as a worldwide positioning system and the main purpose is to provide accurate positioning location at all points on the earth's surface at all times [2 - 5]. It is intended mainly for defense purposes but the civilian community now constitutes the bulk of users. The satellites constellation comprises 24 satellites such that at least 4 satellites are visible everywhere on earth at any time. The orbits are essentially circular at an altitude of about $20,200 \mathrm{~km}$, with orbital inclinations of $63^{\circ}$ and with $12 \mathrm{~h}$ (sidereal time) duration [2 - 5]. In order to provide accurate data and cost effective, a simple and low cost data acquisition system experiment can be used to carry out measurement for different mobile satellite signals. The signal performance of the mobile Satellites (MS) is affected by factors such as ionosphere effect, tree-shadowed, building-shadowed and multipath, but this paper only concentrates on the satellite signals for an open space environment in which the arriving satellite signal does not experience significant fading effect due to trees or building.

Some experiment works have been carried out in some developed countries such as Europe, North America, Japan and Australia [6 - 8]. But little data represents the less developed countries such as Latin America, Africa and Asia. So experiments works are needed in those less develop countries.

Finally a simple and low-cost data acquisition system refers to the experimental setup of the equipments that is the connection formed between the GPS receiver with the computer [9]. The satellite propagation parameters received from the GPS satellite are recorded. The experiment is carried out in an open space and the analysis is then performed to determine the relationship between the signal performance with respect to the elevation and azimuth angles. The simplicity means that the set is simple where we used the simple, readily available and portable GPS receiver connected to the PC for data acquisition. The set up can be used any where we want. While cost effective referred to using the lowcost and readily available GPS receiver that we can come up with the set up as compared to equipment designed specifically for experimental purpose that normally very expensive.

\section{RESEARCH METHODOLGY}

The system design includes developing a simple and lowcost data acquisition system to carry out the measurements for 